\title{
Development of a Segregated Compressible Flow Solver for Turbomachinery Simulations
}

\author{
J. Benajes ${ }^{1}$, J. Galindo ${ }^{1}$, P. Fajardo ${ }^{2}$ and R. Navarro ${ }^{1+}$ \\ ${ }^{1}$ CMT - Motores Térmicos, Universitat Politècnica de València, Camino de Vera S/N, Valencia, 46022, \\ Spain \\ ${ }^{2}$ Bioingeniería e Ingeniería Aeroespacial, Universidad Carlos III de Madrid, Leganés, 28911, Spain
}

†Corresponding Author Email: ronagarl@mot.upv.es

(Received September 10, 2013; accepted January 13, 2014)

\begin{abstract}
A steady multiple reference frame segregated compressible solver and an unsteady sliding mesh one are developed using OpenFOAM ${ }^{\circledR}$ to simulate turbomachinery. For each of the two solvers, governing equations, numerical approach and solver structure are explained. Pressure and energy equation are implemented so as to obtain the best numerical properties, such as the ability to use large time-steps. Sod shock tube test case is used to assess the prediction of compressible phenomena by the transient scheme, which shows proper resolution of compressible waves. Both solvers are used to simulate a turbocharger turbine, comparing their solutions to corresponding ones using ANSYS $^{\circledR}$ Fluent $^{\circledR}$ as a means of validation. The multiple reference frame solver global results quantitatively differ from those computed using ANSYS Fluent, although predicted flow features match. The solution obtained by the sliding mesh solver presents better agreement compared to ANSYS Fluent one.
\end{abstract}

Keywords: CFD, OpenFOAM, Multiple Reference Frame, Sliding Mesh.

\section{NOMENCLATURE}

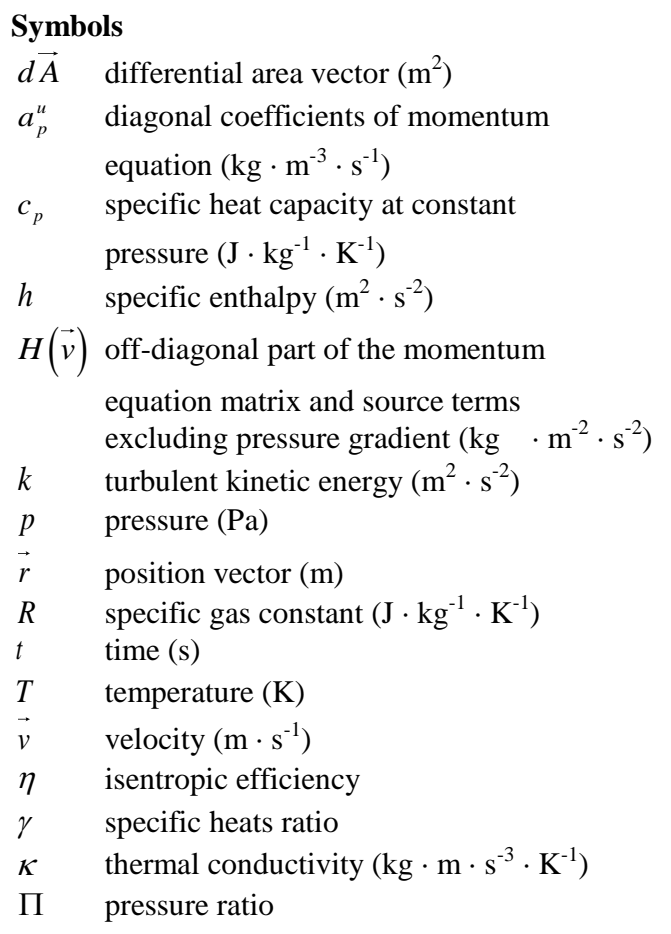

$$
\begin{array}{ll}
\phi_{d} & \text { pseudo-flux }\left(\mathrm{m}^{-1} \cdot \mathrm{s}\right) \\
\psi & \text { compressibility }\left(\mathrm{m}^{-2} \cdot \mathrm{s}^{2}\right) \\
\rho & \text { density }\left(\mathrm{kg} \cdot \mathrm{m}^{-3}\right) \\
\overline{\bar{\tau}} & \text { viscous stress tensor }(\mathrm{Pa}) \\
\vec{\omega} & \text { rotational speed }\left(\mathrm{rad} \cdot \mathrm{s}^{-1}\right)
\end{array}
$$

\section{Sub- and Superscripts}

$0 \quad$ stagnation variable

eff effective value

in inlet value

$f \quad$ values at faces of control volumes

out outlet value

$p \quad$ value for generic point $\mathrm{P}$

$r \quad$ relative

ref reference value

$\begin{array}{ll}\text { Abbreviations } \\ \text { BC } & \text { boundary condition } \\ \text { CFD } & \text { computational fluid dynamics } \\ \text { EOS } & \text { equation of state } \\ \text { GGI } & \text { general grid interface } \\ \text { MRF } & \text { multiple reference frame } \\ \text { SM } & \text { sliding mesh } \\ \text { URF } & \text { under-relaxation factor }\end{array}$




\section{INTRODUCTION}

CFD has become an essential tool in turbomachinery design and analysis, particularly in automotive turbochargers. The flow passing through this type of radial turbomachinery must be considered as compressible, due to the high Mach numbers (Simpson et al. 2009). When simulating compressible flow, there are two types of solvers. The so-called density based solvers can be employed, in which the use of Riemann solvers enhances the ability to capture shocks (Toro 1999). These solvers are thus well-suited for solving hypersonic flow problems (Nair et al. 2010). However, they have a high computational cost, over all when dealing with steady computations, since they rely on a time-marching process. Computation of subsonic/transonic turbomachinery flows can also be carried out by pressure-based segregated solvers, in which a pressure equation is derived from continuity and momentum equations. Since the governing equations are resolved in a sequential fashion, a pressure-velocity coupling method is required, such as the well known SIMPLE (Patankar and Spalding 1972) or PISO (Issa 1986) algorithms.

Another important feature in solvers used to simulate turbomachinery is the strategy to deal with rotor motion. There are two main methods. On the one side, there is the Multiple Reference Frame (MRF) approach, also known as frozen rotor, in which the mesh does not move and the impeller region is modeled with a rotating frame. This technique allows solving a set of steady equations, thus having little computational cost. Several authors have studied the accuracy of this method (Hillewaert and Van den Braembussche 1999; Liu and Hill 2000; Zheng et al. 2010). The general agreement is that MRF is not the most appropriate approach for radial turbomachinery simulations, especially when predicting flow features at offdesign conditions. However, due to its low computational cost, it is useful as a first hint of the turbomachinery performance or as a means of initialization for more complex methods. On the other side, the Sliding Mesh (SM) approach considers the unsteady equations, rotating the impeller mesh at every time-step.

Reaching a periodic state requires 10 to 100 times more computational cost than obtaining the converged steady solution with MRF. However, transient features are resolved, which is of importance when simulating turbomachinery at offdesign operating conditions (Galindo et al. 2013a). The SM method has been proved to give good results (Hellstrom 2010; Guo et al. 2007; Chen et al. 2008).

One of the codes whose use is steadily increasing is OpenFOAM. OpenFOAM (OpenCFD Ltd 20042013) is an open source toolbox for the solution of continuum mechanics problems. OpenFOAM has been used for several incompressible turbomachinery simulations. Auvinen et al. (2009) conducted a numerical study of a single-channel pump, comparing CFD results against experimental data. Transient simulation were performed using a sliding mesh approach with the aid of a General Grid Interface (GGI), a tool developed by Beaudoin and Jasak (2008). The effect of mesh density, turbulence models, time-step, and length of inlet duct on overall parameters such as hydrodynamic head, shaft power or efficiency was checked. Moreover, velocity profiles predicted by the different numerical configurations are compared with Laser Doppler Velocimetry measurements. Petit et al. (2009) compared frozen rotor and sliding grid 2D simulations of the ERCOFTAC centrifugal pump against experimental data obtained by Ubaldi et al. (1996). GGI variants and additional turbomachinery simulations performed with OpenFOAM can be found in Jasak (2011).

Regarding compressible flow turbomachinery simulations using OpenFOAM, Borm et al. (2011a,b; 2012) developed and tested several density-based solvers with MRF and SM capabilities, which are available at one branch of the Extend Project Borm (2012). Mangani et al. (2007) developed a pressure-based segregated compressible solver in OpenFOAM, using a pressure corrector equation. However, rotor motion methods were not discussed. Mangani et al. (2012) investigated the effect of turbulence models on the prediction of centrifugal compressor global variables and local flow field. Gröschel et al. (2012) used an improved version of this solver with a MRF approach to optimize a high pressure ratio centrifugal compressor, although the implementation of the MRF method is not described.

The main advantages of OpenFOAM are that it is free of charge, so it does not have any license code nor license manager problems, and it is an open code, which allows one to modify almost every single detail of it. However, since the code is relatively young, it lacks some capabilities and some existing ones have not been thoroughly validated. For instance, there are no compressible pressure based solvers available in OpenFoam having a built-in rotor motion capability, which is a must in turbomachinery applications, and allowing to perform computations with large time-step size. The objective of this paper is therefore to develop compressible pressure-based segregated solvers for turbomachinery flow simulations using OpenFOAM, with both MRF and SM approaches. The solvers have been implemented in OpenFOAM-1.6-ext.

For each of the two solvers, corresponding governing equations will be first presented and the solver structure will be explained. Then, the solver will be used to simulate a turbocharger turbine, comparing the solution obtained to the one computed using ANSYS Fluent.

\section{MULTIPLE REFERENCE FRAME}

The governing equations in a rotating reference frame, using the absolute velocity formulation, can be expressed as: 


$$
\begin{aligned}
& \nabla \cdot\left(\rho \vec{v}_{r}\right)=0 \\
& \nabla \cdot\left(\rho \vec{v}_{r} \vec{v}\right)+\rho(\vec{\omega} \wedge \vec{v})=-\nabla p+\nabla \cdot \overline{\bar{\tau}} \\
& \nabla \cdot\left[\rho \vec{v}_{r} h_{0}+p(\vec{\omega} \wedge \vec{r})\right]=\nabla \cdot\left(\kappa_{e f f} \nabla T+\overline{\bar{\tau}} \cdot \vec{v}\right) \\
& p=\rho R T=\frac{\rho}{\psi}
\end{aligned}
$$

where total enthalpy is defined as:

$$
h_{0}=h+\frac{|\vec{v}|^{2}}{2}+k \text {. }
$$

The differences between Eq. (1) and the ordinary Navier-Stokes equations are that the advective terms are computed with a relative flux $\left(\vec{v}_{r}=\vec{v}-\vec{\omega} \wedge \vec{r}\right)$ and there are new terms due to non inertial effects.

It is important to highlight that energy equation has been considered using total enthalpy because it provides a conservative formulation. Since a segregated approach is sought, the pressure equation is derived using continuity and momentum ones following the work by Jasak (2006), resulting in Eq. (3):

$\sum_{f} \phi_{d, f} p_{f}=\nabla \cdot\left(\rho\left(a_{p}^{u}\right)^{-1} \nabla p\right)$.

Left hand side of Eq. (3) has been integrated over a control volume using a linearized and discretized form of Gauss' theorem. Since Eq. (3) is not a pressure corrector equation, implicit underrelaxation can be performed, thus improving the linear solver stability (Ferziger and Peric 2002), and the restrictions on boundary conditions described by Mangani (2008) are not present. In Eq. (3) a pseudo-flux, $\phi_{d}$, is used as presented by Jasak
(1996). $\phi_{d}$ is computed as shown in Eq. (4):

$\phi_{d}=\psi\left(\left(a_{p}^{u}\right)^{-1} H(\vec{v})\right) d \vec{A}$,

in which the pseudo-velocity employed, $\left(a_{p}^{u}\right)^{-1} H(\vec{v})$, does not carry the pressure gradient contribution. The pseudo-velocity field can be obtained rearrainging the momentum equation, as shown in Eq. (6):

$a_{p}^{u} \overrightarrow{v_{p}}=H(\vec{v})-\nabla p$,

where the terms of the equation have been separated in the diagonal terms, $a_{p}^{u} \overrightarrow{v_{p}}$, and the off-diagonal part and rest of the sources of the equation excluding the pressure gradient, represented by the operator $H(\vec{v})$. Additionally, since it is a MRF solver, the flux in the rotating domain has to be relative to the rotating reference frame, as:

$$
\phi_{d, r}=\psi\left(\left(a_{p}^{u}\right)^{-1} H(\vec{v})-\vec{\omega} \wedge \vec{r}\right) d \vec{A}
$$

The solver structure is sketched in Fig. 1. Following the SIMPLE approach, a momentum predictor comes first, in which velocity equation is defined, implicitly under-relaxed and solved. Then, the pseudo-flux is computed. However, since implicit under relaxation has changed the matrix coefficients of momentum equation, an update of the equation coefficients is required to erase this effect and ensure that the solution fulfils continuity. This is something not done by the compressible solvers currently available in OpenFOAM. Afterwards, pressure equation is assembled, implicitly relaxed and solved.

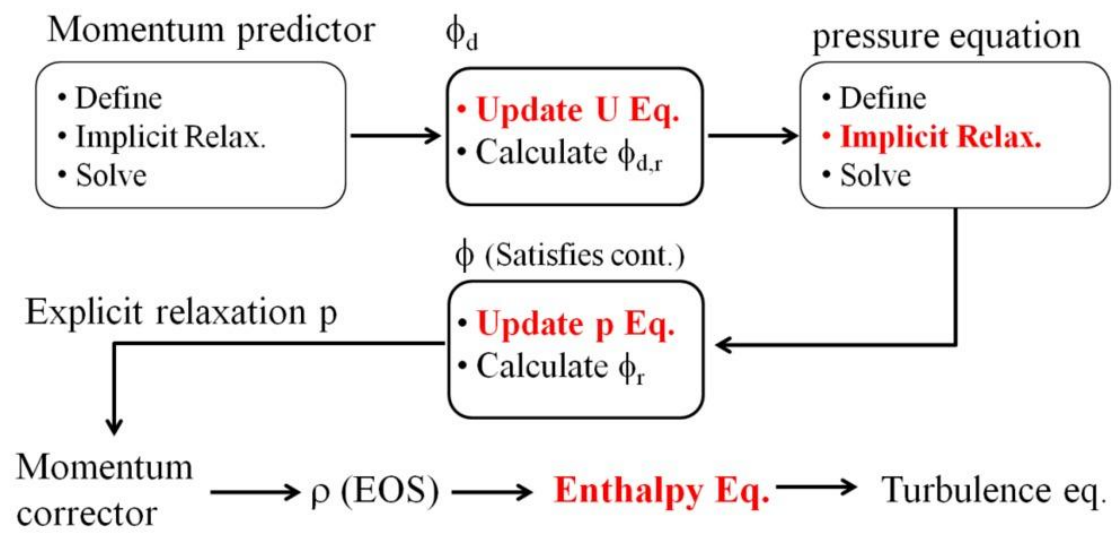

Fig. 1. Flowchart of the proposed MRF solver. Major changes regarding already available OpenFOAM solvers are highlighted in red

Since the equations are implemented in a conservative form, in which continuity is implicitly considered, it is important that the calculated fluxes satisfy continuity. In order to obtain a set of face fluxes that fulfills this requirement, two things must be taken into account: the coefficient matrix of pressure equation should be updated before obtaining the flux, for the same reason as in momentum equation, and the implicit URF should be close to 1 . Then, pressure field is explicitly relaxed and velocity is corrected with the new pressure contribution.

Finally, density is obtained using the equation of state (EOS) and enthalpy and turbulent equations 
are solved. After that, a new iteration is performed. Regarding enthalpy equation, it has been placed after the pressure-velocity coupling to have a consistent set of pressure and velocity fields and fluxes when solving it. If the fluid cell considered belongs to the rotor, the corresponding non-inertial terms must be added prior to solving momentum and energy equations and the fluxes should be made relative to the rotating reference frame after its computation.

The developed solver was used to simulate the variable geometry turbine analyzed by Galindo et al. (2013b) under different operating conditions, providing good convergence behavior. The solution obtained by this solver is compared to the one computed using ANSYS Fluent. The same setup, described in Table 1, is used in both codes. First order discretization schemes have been chosen for the current simulations. This selection is based on the fact that the implementation of $1^{\text {st }}$ order schemes is unequivocal while the implementation of a $2^{\text {nd }}$ order scheme could have different approaches in each code, and the goal of the simulations is not accuracy of the solution but comparability across codes.
Table 1 MRF configuration parameters

\begin{tabular}{|l|l|}
\hline $\begin{array}{l}\text { Discretization } \\
\text { schemes }\end{array}$ & $1^{\text {st }}$ order \\
\hline Inlet BC & $\begin{array}{l}\text { Mass flow }(0.065,0.075, \\
0.085,0.095 \mathrm{~kg} / \mathrm{s}), \\
\text { total temperature }(664 \mathrm{~K})\end{array}$ \\
\hline Outlet BC & Static pressure $(101325 \mathrm{~Pa})$ \\
\hline $\begin{array}{l}\text { Wall heat transfer } \\
\text { model }\end{array}$ & Adiabatic \\
\hline Turbulence model & RNG k- $\varepsilon$ \\
\hline Rotational speed & $18953 \mathrm{rad} / \mathrm{s}$ \\
\hline $\begin{array}{l}\text { Thermal properties } \\
\left(\mathrm{c}_{\mathrm{p}}, \kappa, \text { etc. }\right)\end{array}$ & $\begin{array}{l}\text { Constant values for dry air at } \\
664 \mathrm{~K}\end{array}$ \\
\hline
\end{tabular}

The mesh used by both codes is the one represented in Fig. 2, with 1.2 million cells. A mesh independence analysis was performed to determine its influence on the solution. It was observed that doubling the total number of cells, the solution changed about a $0.5 \%$ in pressure drop and a $2 \%$ in torque. Since the objective of this paper is to compare solvers, the additional computational cost of increasing the number of cells in order to obtain the most accurate solution was not justified.

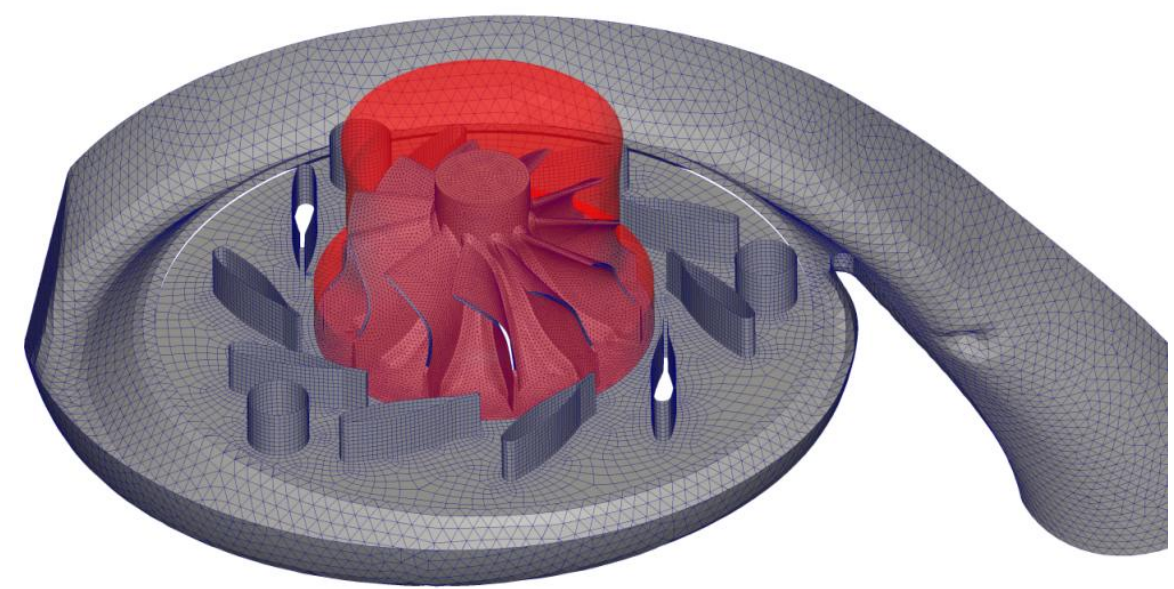

Fig. 2. 1.2 million cells mesh used for solvers comparison. The rotor region has been marked in red.

To compare the global performance of the turbine predicted by both codes, flow capacity and efficiency curves are computed using the results from the 4 simulated operating conditions. In order to compute flow capacity of the turbine, corrected mass flow is defined as shown in Eq. (8):

$$
\dot{m}_{i n}^{*}=\frac{\dot{m}_{i n} \sqrt{\frac{T_{i n, 0}}{T_{r e f}}}}{\frac{p_{i n, 0}}{p_{r e f}}},
$$

in which $p_{\text {ref }}=101325 \mathrm{~Pa}$ and $T_{\text {ref }}=288.15 \mathrm{~K}$, and total-to-static pressure ratio is calculated

following Eq. (9):

$\Pi_{t, s}=\frac{p_{\text {in }, 0}}{p_{\text {out }}}$.

Left side of Fig. 3 shows the flow capacity predicted by the 2 codes, obtained from the 4 different MRF cases. The computed values differ up to $10 \%$. 

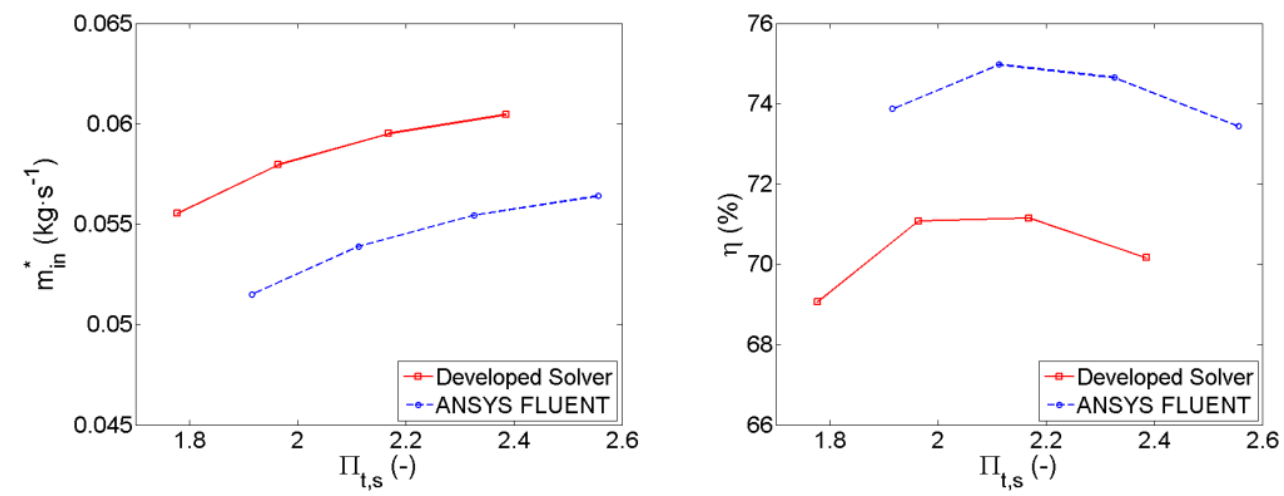

Fig. 3. Flow capacity predicted by ANSYS Fluent (blue) and the developed MRF solver (red)

Isentropic turbine efficiency is calculated taking into account the perfect gas hypothesis, as:

$$
\eta=\frac{\dot{W}}{\dot{W}_{i s}}=\frac{T_{i n, 0}-T_{o u t, 0}}{T_{i n, 0}\left(1-\Pi_{t, s}^{\frac{1-\gamma}{\gamma}}\right)} .
$$

Right side of Fig. 3 presents the isentropic turbine efficiency against pressure ratio. Maximum efficiency point is located at a similar expansion ratio, although the developed solver predicted efficiencies are about $6 \%$ less than ANSYS Fluent ones.

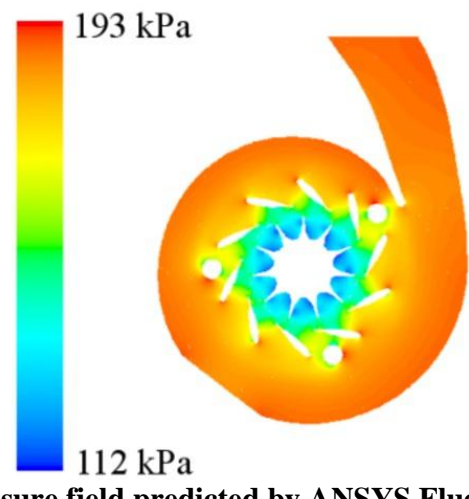

Fig. 4. Pressure field predicted by ANSYS Fluent (left) and the developed MRF solver (right) for the $0.065 \mathrm{~kg} / \mathrm{s}$ case.

\section{SLIDING MESH}

Sliding mesh governing equations (Eq. (11)) are similar to Eq. (1) in the fact that relative fluxes are employed. However, the corresponding temporal terms are not dropped and there are no non-inertial extra terms:

$$
\begin{aligned}
& \frac{\partial \rho}{\partial t}+\nabla \cdot\left(\rho \vec{v}_{r}\right)=0 \\
& \frac{\partial \rho \vec{v}}{\partial t}+\nabla \cdot\left(\rho \vec{v}_{r} \vec{v}\right)=-\nabla p+\nabla \cdot \overline{\bar{\tau}} \\
& \frac{\partial \rho h_{0}}{\partial t}+\nabla \cdot\left(\rho \vec{v}_{r} h_{0}\right)=\frac{\partial p}{\partial t}+\nabla \cdot\left(k_{e f f} \nabla T+\overline{\bar{\tau}} \cdot \vec{v}\right) \\
& p=\rho R T=\frac{\rho}{\psi}
\end{aligned}
$$

The case in which the imposed mass flow is $0.065 \mathrm{~kg} / \mathrm{s}$ is studied in more detail. Fig. 4 shows a comparison between the pressure fields at the stator predicted by both codes for the aforementioned case. The scale has been deliberately set with different limits. In this way, one can see that the flow features are very similar, although the pressure drop is clearly underpredicted by the proposed solver.

Fig. 5 depicts the velocity field at the turbine stator predicted by ANSYS Fluent (left side) and the developed solver (right side) for the same case. Except at the proximity of the bolts, velocity fields are almost identical.

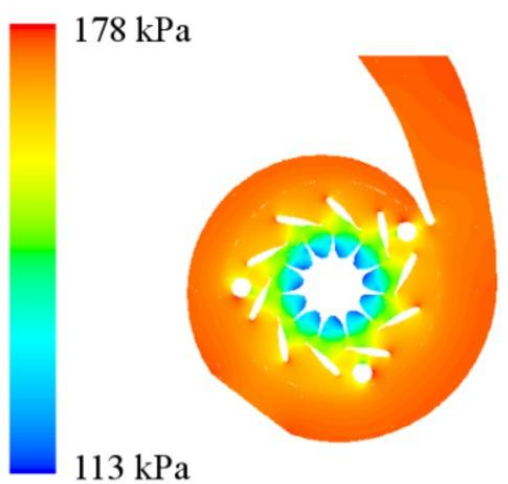

The pressure equation is again derived using continuity and momentum ones, resulting in Eq. (11):

$$
\frac{\partial \psi p}{\partial t}+\sum_{f} \phi_{d, f} p_{f}=\nabla \cdot\left(\rho\left(a_{p}^{u}\right)^{-1} \nabla p\right) .
$$

The pseudo-flux $\phi_{d}$ used in Eq. (12) is the one defined by Eq. (5) (or Eq. (7) if the considered cell is in the rotating domain). As in Eq. (3), the convective term is presented in a discretized form.

The solver structure is sketched in Fig. 6. Only the major differences respect to the one shown in Fig. 1 will be pointed out. When starting a new time-step, the mesh is correspondingly rotated and continuity equation is firstly solved. Then, the so-called PIMPLE loop is applied. Momentum predictor is 
followed by a number of pressure equation and momentum corrector loops, known as inner loops. Afterwards, density is updated and enthalpy and turbulent equations are solved. The whole set of equations, excluding continuity, form an outer loop which is repeated a predefined number of times to overcome the segregated approach. After that, a new time-step can be performed.
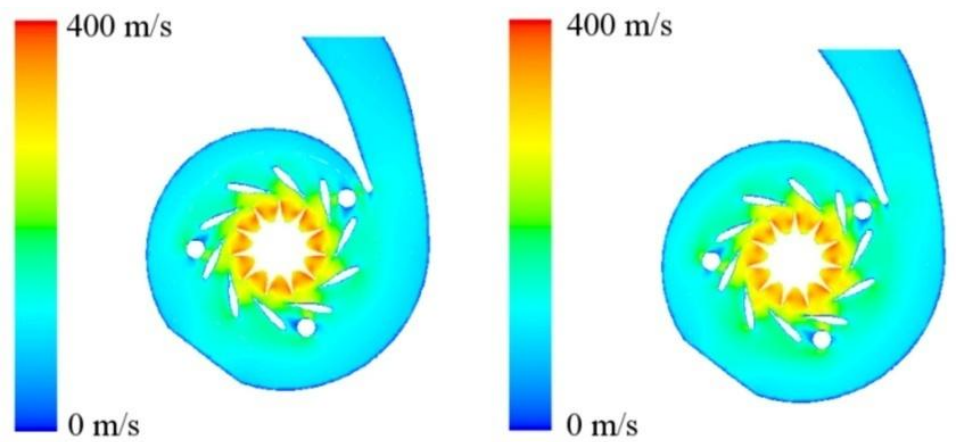

Fig. 5. Velocity field predicted by ANSYS Fluent (left) and the developed MRF solver (right) for the $0.065 \mathrm{~kg} / \mathrm{s}$ case.

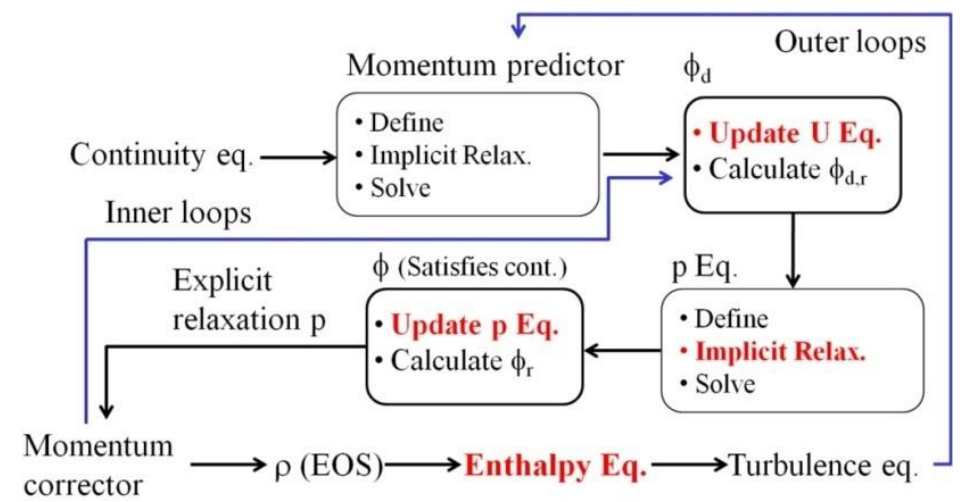

Fig. 6. Flowchart of the proposed SM solver. Major changes regarding already available OpenFOAM solvers are highlighted in red.

Sod shock tube test case (Sod 1978) is used to assess the ability of the transient solver to capture compressible phenomena. The test has been set up in the same way as in Galindo et al. (2011). A 2-mlong tube is divided by a membrane into two separate regions: the domain at the left side of the membrane is initially set at a pressure of 3 bar and a temperature of $293 \mathrm{~K}$, while the right side is set at a pressure of 1 bar and a temperature of $293 \mathrm{~K}$. At time zero this membrane is eliminated and the flow inside the tube starts to evolve.

The results obtained by the developed solver are presented in Fig. 7 in terms of temperature, velocity, pressure and density axial profiles at time $2 \mathrm{~ms}$ compared against analytical solution. Contact and shock discontinuities and rarefaction wave are captured by the solver. An undershoot of the profiles is observed, but it disappears as long as the number of cells is increased, achieving a perfect match with analytical solution (not shown here).

In order to check the sliding mesh capability of the developed solver, it is used to simulate transient behavior of the turbine at lowest mass flow operating conditions (see Table 1). At every timestep 10 outer loops are performed, causing the residuals to drop several orders of magnitude and thus ensuring a good convergence. The solution obtained by this solver is compared to the one computed using ANSYS Fluent. The mesh and setup is the same as the one used in section 2. The time-step was chosen so that the mesh turns $1^{\circ}$ per time-step.

Fig. 8 shows a comparison between the pressure field at the stator predicted by ANSYS Fluent (left side) and the proposed solver (right side). Both solutions are very similar, although the developed solver pressure at inlet is slightly higher. Fig. 9 depicts the velocity field at the stator computed by the two codes for the same conditions. Again, the velocity fields are almost identical.

Fig. 10 presents the torque evolution predicted by both codes. Blade passing frequency is properly captured by the two codes, although the developed solver predicts oscillations which amplitude is $15 \%$ greater than the one obtained using FLUENT. Torque mean values differ in $3.5 \%$. 

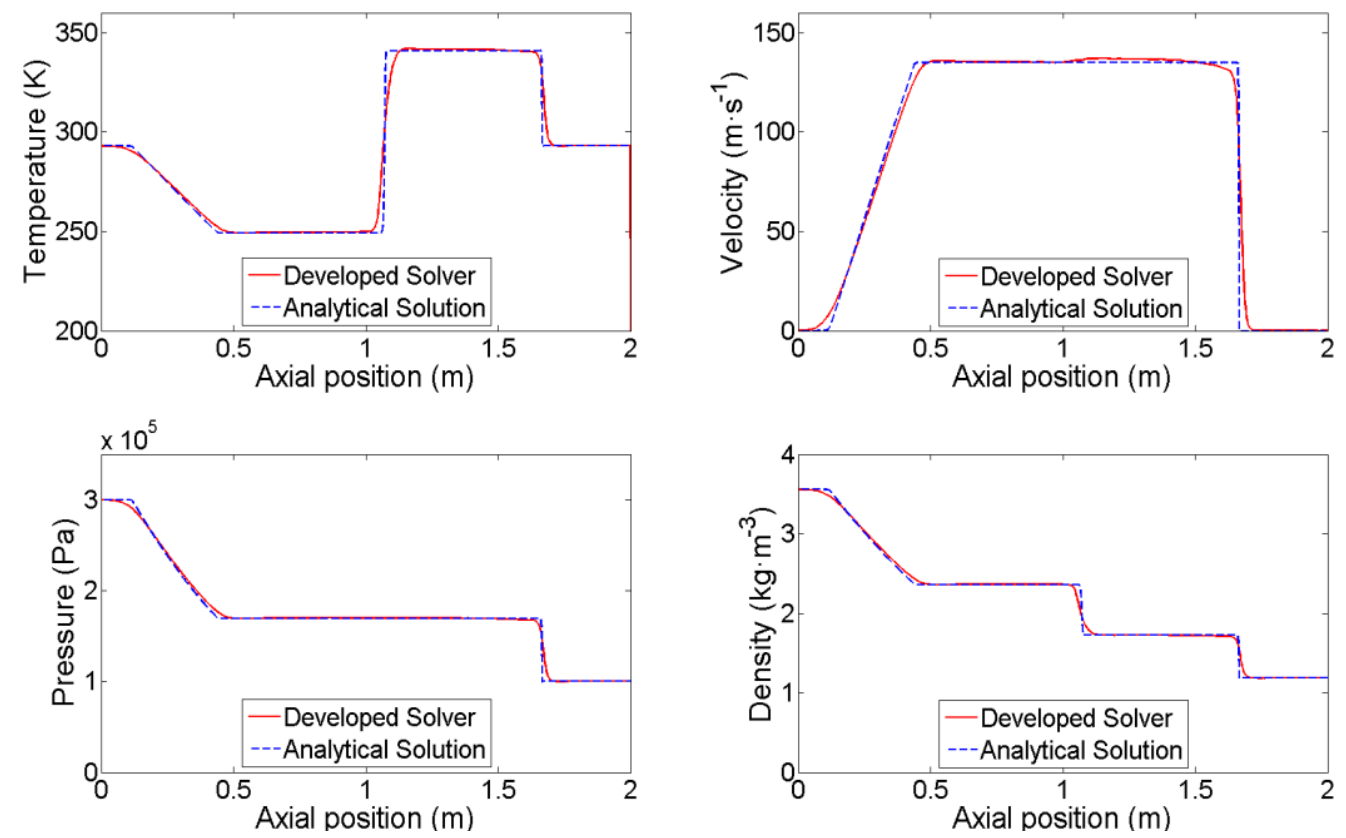

Fig. 7. Comparison of developed solver and analytical flow profiles at time $2 \mathrm{~ms}$ for Sod shock tube test case. Temperature (top left), velocity (top right), pressure (bottom left) and density (bottom right).
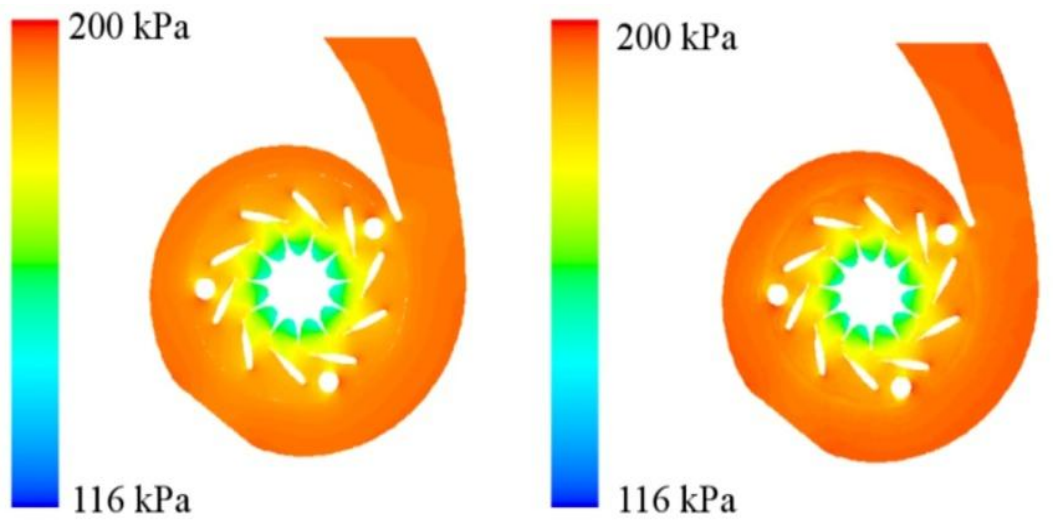

Fig. 8. Pressure field predicted by ANSYS Fluent (left) and the developed SM solver (right).
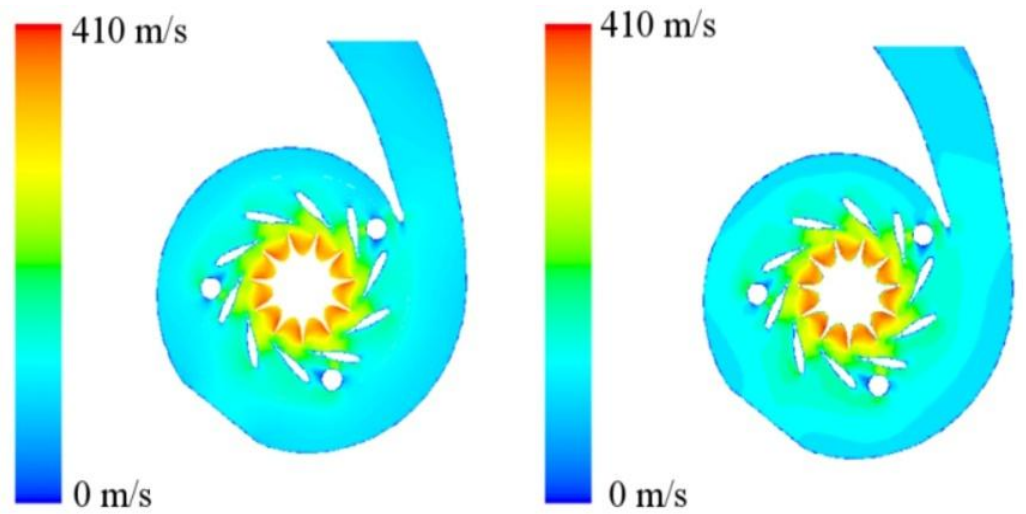

Fig. 9. Velocity field predicted by ANSYS Fluent (left) and the developed SM solver (right). 


\section{CONCLUSIONS}

In this paper, a MRF compressible solver and a SM one have been developed using OpenFOAM to simulate turbomachinery. Pressure equation has been derived following the work by Jasak (1996), instead of using a pressure corrector equation. In this way, implicit under-relaxation can be performed, with the corresponding increase of linear solver stability and avoiding restrictions on boundary conditions. Energy equation has been implemented using total enthalpy because it provides a conservative formulation.

The structure of the SM solver, which enables computations with a large time-step size due to the existence of inner and outer loops and underrelaxation, and the rotor motion capability make the developed solver suitable for performing automotive turbocharger simulations, not having currently an equivalent pressure based segregated compressible OpenFOAM solver.

Both solvers have been used to simulate a turbocharger turbine, showing good convergence behavior. Their solutions have been compared to corresponding ones computed using ANSYS Fluent, as a means of validation. The MRF solver predicts proper flow features. However, it still needs some work since the provided values can differ up to $10 \%$ compared to ANSYS Fluent ones. The SM solver obtains a more accurate solution, even though some differences exist compared to ANSYS Fluent. Compressible phenomena prediction by the transient solver is confirmed by Sod shock tube test case.

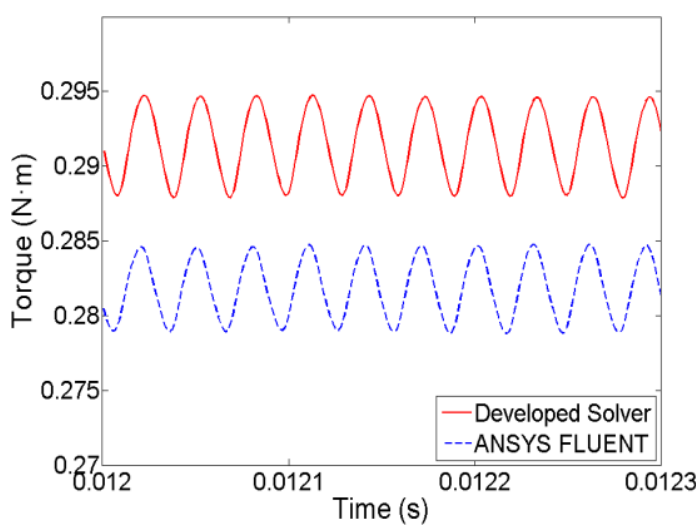

Fig. 10. Evolution of torque with time computed by ANSYS Fluent (blue) and the developed SM solver (red).

\section{ACKNOWLEDGEMENTS}

The authors are indebted to the Spanish Ministerio de Economía y Competitividad through Project TRA 2010-16205.

\section{REFERENCES}

Auvinen, M., J. Ala-Juusela, N. Pedersen, and T. Siikonen (2010). Time-Accurate Turbomachinery Simulations with Open-
Source CFD; Flow Analysis of a SingleChannel Pump with OpenFOAM. In ECCOMAS CFD 2010.

Beaudoin, M. and H. Jasak (2008, December). Development of a Generalized Grid Interface for Turbomachinery Simulations with OpenFOAM. In Open Source CFD International Conference. Berlin, Germany.

Borm, O., B. Balassa, and H.-P. Kau (2011a). Comparison of Different Numerical Approaches at the Centrifugal Compressor RADIVER. In $20^{\text {th }}$ ISABE Conference, Number ISABE-2011-1242. International Society for Airbreathing Engines.

Borm, O., A. Jemcov, and H. P. Kau (2011b). Density Based Navier Stokes Solver for Transonic Flows. In $6^{\text {th }}$ OpenFOAM Workshop.

Borm, O. (2012). Density Based Turbo Solvers. URL: $\quad$ http://sourceforge.net/p/openfoamextend/DensityBasedTurbo/ci/master/tree/.

Borm, O. and H. P. Kau (2012). Unsteady Aerodynamics of a Centrifugal Compressor Stage - Validation of Two Different CFD Solvers. In Proceedings of ASME Turbo Expo 2012, Number GT2012-69636. ASME.

Chen, H., S. Guo, X. C. Zhu, Z. H. Du, and S. Zhao (2008). Numerical Simulations of Onset of Volute Stall Inside a Centrifugal Compressor. In Proceedings of ASME TurboExpo 2008: Power for Land, Sea and Air. ASME.

Ferziger, J. H. and M. Peric (2002). Computational Methods for Fluid Dynamics ( $3^{\text {rd }}$ ed.). Springer.

Galindo, J., A. Tiseira, P. Fajardo, and R. Navarro (2011). Coupling methodology of 1D finite difference and 3D finite volume CFD codes based on the Method of Characteristics. Mathematical and Computer Modelling 54 (78), 1738-1746.

Galindo, J., P. Fajardo, R. Navarro, and L. M. García-Cuevas (2013a). Characterization of a radial turbocharger turbine in pulsating flow by means of CFD and its application to engine modeling. Applied Energy 103, 116-127.

Galindo, J., S. Hoyas, P. Fajardo, and R. Navarro (2013b). Set-up analysis and optimization of 
CFD simulations for radial turbines. Engineering Applications of Computational Fluid Mechanics 7(4), 441-460.

Gröschel, E., B. Rembold, L. Mangani, and E. Casartelli (2012). AN OBJECT-ORIENTED CFD CODE FOR OPTIMIZATION OF HIGH PRESSURE RATIO COMPRESSORS. In Proceedings of ASME Turbo Expo 2012, Number GT2012-68708. ASME.

Guo, Q., H. Chen, X. C. Zhu, Z. H. Du, and Y. Zhao (2007). Numerical simulations of stall inside a centrifugal compressor. Proceedings of the Institution of Mechanical Engineers, Part A: Journal of Power and Energy 221(5), 683-693.

Hellström, F. (2010). Numerical computations of the unsteady flow in turbochargers. Ph. D. thesis, Royal Institute of Technology KTH Mechanics.

Hillewaert, K. and R. Van den Braembussche (1999). Numerical simulation of impellervolute interaction in centrifugal compressors. Journal of Turbomachinery 121, 603.

Issa, R. I. (1986). Solution of the implicitly discretised fluid flow equations by operatorsplitting. Journal of Computational physics 62(1), 40-65.

Jasak, H. (1996, June). Error Analysis and Estimation for the Finite Volume Method with Applications to Fluid Flows. Ph. D. thesis, Imperial College of Science, Technology and Medicine.

Jasak, H. (2006-2007). Numerical Solution Algorithms for Compressible Flows (Lecture notes). Faculty of Mechanical Engineering and Naval Architecture.

Jasak, H. and M. Beaudoin (2011). OpenFOAM Turbo Tools: From General Purpose CFD to Turbomachinery Simulations. In ASMEJSME-KSME Joint Fluids Engineering Conference, Number AJK2011-05015. ASME.

Liu, Z. and D. Hill (2000). Issues surrounding multiple frames of reference models for turbocompressor applications. In Fifteenth International Compressor Engineering Conference. Purdue University.

Mangani, L., C. Bianchini, A. Andreini, and B.
Facchini (2007). Development and validation of a $\mathrm{C}++$ object oriented CFD code for heat transfer analysis. In ASME-JSME 2007 Thermal Engineering and Summer Heat Transfer Converence.

Mangani, L. (2008). Development and Validation of an Object Oriented CFD Solver for Heat Transfer and Combustion Modeling in Turbomachinery Applications. Ph. D. thesis, Università degli Studi di Firenze, Florence, Italy.

Mangani, L., E. Casartelli, and S. Mauri (2012). Assessment of Various Turbulence Models in a High Pressure Ratio Centrifugal Compressor With an Object Oriented CFD Code. Journal of turbomachinery 134(6).

Nair, P., T. Jayachandran, M. Deepu, B.P. Puranik and U.V. Bhandarkar (2010). Numerical Simulation of Interaction of Sonic Jet with High Speed Flow over a Blunt Body using Solution Mapped Higher Order Accurate AUSM+-UP Based Flow Solver. Journal of Applied Fluid Mechanics 3(1) 15-23.

OpenCFD Ltd (2004-2013). OpenFOAM. URL: http://www.openfoam.com/.

Patankar, S. V. and D. B. Spalding (1972). A calculation procedure for heat, mass and momentum transfer in three-dimensional parabolic flows. International Journal of Heat and Mass Transfer 15(10), 1787-1806.

Petit, O., M. Page, M. Beaudoin, and H. Nilsson (2009). The ERCOFTAC Centrifugal Pump OpenFOAM Case-Study. In $3^{\text {rd }}$ IAHR International Meeting of the Workgroup on Cavitation and Dynamic Problems in Hydraulic Machinery and Systems, Brno, Czech Republic, October, Volume 14, pp. 16.

Simpson, A. T., S. W. T. Spence, and J. K. Watterson (2009). A comparison of the flow structures and losses within vaned and vaneless stators for radial turbines. Journal of Turbomachinery 131, 031010.

Sod, Gary A. (1978). A survey of several finite difference methods for systems of nonlinear hyperbolic conservation laws. Journal of Computational Physics 27(1), 1-31.

Toro, E. (1999). Riemann solvers and numerical methods for fluid dynamics: a practical 
J. Benajes et al. / JAFM, Vol. 7, No. 4, pp. 673-682, 2014.

introduction ( $2^{\text {nd }}$ ed.). Springer.

Ubaldi, M., P. Zunino, G. Barigozzi, and A. Cattanei (1996). An experimental investigation of stator induced unsteadiness on centrifugal impeller outflow. Journal of turbomachinery $118,41$.
Zheng, X. Q., J. Huenteler, M. Y. Yang, Y. J. Zhang, and T. Bamba (2010). Influence of the volute on the flow in a centrifugal compressor of a high-pressure ratio turbocharger. Proceedings of the Institution of Mechanical Engineers, Part A: Journal of Power and Energy 224(8), 1157-1169. 